\title{
Design of the Large Time-Delay Temperature Control System for a Large-Scale Chicken Farm
}

\author{
Cunming Zou \\ The department of electrical engineering \\ Dalian institute of science and technology \\ Dalian, China \\ e-mail: cunming2004@163.com \\ Tingting Yao \\ The department of electrical engineering \\ Dalian institute of science and technology \\ Dalian, China \\ e-mail: yaotingting816@126.com
}

\author{
Zhichao Xu \\ The department of electrical engineering \\ Dalian institute of science and technology \\ Dalian, China \\ e-mail: xugong20060883@126.com \\ Zhao Xu \\ The department of electrical engineering \\ Dalian institute of science and technology \\ Dalian, China \\ e-mail: ffx820819@163.com
}

\begin{abstract}
Aiming at discussing the important issue of large time-delay temperature control of large-scale chicken farms, a large time-delay model of large-scale chicken house temperature control is firstly established. Through the large time-delay model of the large-scale chicken farm temperature control, the traditional PID temperature control, differential forward PID and Smith predictor PID control algorithm should be used respectively on the temperature control of the large-scale chicken farm. It makes the temperature of the large chicken farm increase fast, and meets the expected temperature stably. Through the analysis and comparison of the three kinds of simulation results, the validity of the Smith predictor method for the large time-delay temperature control system is verified.
\end{abstract}

Keywords- large-scale chicken house temperature; traditional PID ; differential forward; Smith predictor

\section{INTRODUCTION}

Large-scale intelligent chicken farms have high intelligent degree, low production cost, fast breeding, high survival rate and so on. Due to those advantages, the intelligent large-scale farms are applied extensively [1][2]. By the 21 st Century, the rapid development of intelligent control technology and other related fields has laid a solid foundation for the wide spread use of the intelligent control and monitoring system in the field of large-scale breeding industry[3]. At present, the research on the intelligent large-scale farms in foreign countries is ahead of China, and it has already had a more mature example, and has been widely used. Among them, the large-scale intelligent chicken farms have become a hot spot of research in recent years, which attracts more and more researchers' attention, and expands its application scope in China and abroad[4].

The temperature of the chicken house, water quality and ventilation condition have a great influence on the growth of chicks. And it also exists some of these problems on the large farm system, so a part of research works on the environmental monitoring of large intelligent farming field[5][6], and another part of the research tends to control and optimize some index. In order to improve water quality, some people like Zhao Li Ming have studied the development of livestock automatic drinking device for years, on the basis of which they designed a spoon chicken with automatic drinking appliance and won the national patent in 2002[7][8]. Since then some people have made a continuous effort to improve and optimize the automatic drinking device for poultry [9], studying how to select a more suitable device [10]. And in order to solve the problem of ventilation farms, literature[11] made smartflow intelligent ventilation system used in the pig farm. Literature[12] is the research of modern poultry floor ventilation system. But the study for the problem of the chicken farm temperature with large time-delay chicken temperature control is less, while the text is to study this problem.

According to the style of the building, the large intelligent chicken farm is divided into two kinds: one is the construction of conventional way, with the building of the hollow brick and insulation board is built, this approach is more commonly used, and the manufacturing cost is low, but the sealing is poor, and it will have a greater delay for farm temperature control. The other is totally-enclosed construction way, which has better sealing but poor air circulation, so it need to change the wind regularly, which greatly increases the cost of production. Generally speaking, this kind of construction's cost is 3-4 times higher than conventional construction. Therefore most of us use the first construction method, but it sets us high demand for the accuracy and speed of the temperature control.

This paper, firstly, through the experimental method of large-scale intelligent chicken farm temperature field control system model, and on this basis, the system using a variety of methods for ambient temperature control, and 
through the Simulink simulation proving the PID control method, differential forward control method and Smith predictor, illustrates the effectiveness of large-scale intelligent chicken farms temperature control.

\section{MODEL OF LARGE TIME-DELAY TEMPERATURE OF LARGE-SCALE CHICKEN FARMS}

At present, we mostly adopts PID temperature control on the temperature control of large-scale intelligent farm, heating equipment and external environment and the way of temperature control asks a great deal for the large-scale intelligent chicken farms. For large intelligent farm when heating using the heating boiler, it often needs a long time to reach the desired temperature value, and during also the heating process, it often fluctuates within the desired temperature range, which has a great influence on the chick growth. Especially in the winter, with the low surrounding environment, slow warming, as long as the temperature difference between 2 degrees, within 15 minutes chicks will get sick, greatly increasing the mortality of chicks. The for the large-scale intelligent of chicken control delay, design method of large scale chicken farm temperature controller based on Smith predictor, and gives the simulation of PID control and differential advanced control algorithm is proposed, and with comparing methods proves that the Smith predictor design can greatly improve the speed and accuracy of the control object.

Above all, the temperature environment of large intelligent chicken farms is complex, and it exists many factors which affect the temperature. Therefore, we set up time-delay model with adopting the experimental method. And through the experimental method, the first-order inertia plus pure delay model is established, as follows:

$$
G(\mathrm{~s})=\mathrm{K}_{P} \mathrm{G}_{\mathrm{P}} \mathrm{e}^{-\tau s}=K_{P} \frac{1}{T_{1} s+1} \mathrm{e}^{-\tau s}
$$

Design of large time-delay temperature controller for large chicken farms

A. Design of PID controller for large delay temperature of large chicken farms

PID temperature controller for large chicken farms is a very common method, according to the standard form of PID control, the temperature of the expected value of $F(t)$ is:

(2)

$$
\mathrm{F}(\mathrm{t})=\mathrm{K}_{\mathrm{P}} \mathrm{e}(\mathrm{t})+K_{I} \int e(\mathrm{t}) \mathrm{dt}+K_{D} \frac{d e(\mathrm{t})}{d t}
$$

In the formula:

$\mathrm{F}(\mathrm{t})$ expresses $\mathrm{t}$ moment's temperature control signal.

$\mathrm{K}_{\mathrm{P}}$ expresses scaling factor.

$\mathrm{e}(\mathrm{t})$ expresses $\mathrm{t}$ moment's deviation of the actual temperature and the desired temperature.

$\mathrm{K}_{\mathrm{I}}$ expresses the integral time, the ratio of the proportional coefficient and the integral time constant.

$\mathrm{K}_{\mathrm{D}}$ expresses the rate time, the product of proportional coefficient and rate time constant.

According to the model expression of the large-scale intelligent chicken farms large delay temperature(1), the structure of temperature control system simulation diagram is as shown in Figure 1, $\mathrm{zd}$ is desired temperature. Here the temperature value which we firstly expect to take is 20 degrees, and we take $\mathrm{K}_{\mathrm{P}}=5, \mathrm{~T}_{1}=10$;

In the process of simulation, we get the parameters of PID by using the conventional PID tuning method:

$$
\mathrm{K}_{\mathrm{P}}=0.4352 \quad \mathrm{~K}_{\mathrm{I}}=0.0047 \quad \mathrm{~K}_{\mathrm{D}}=0.9
$$

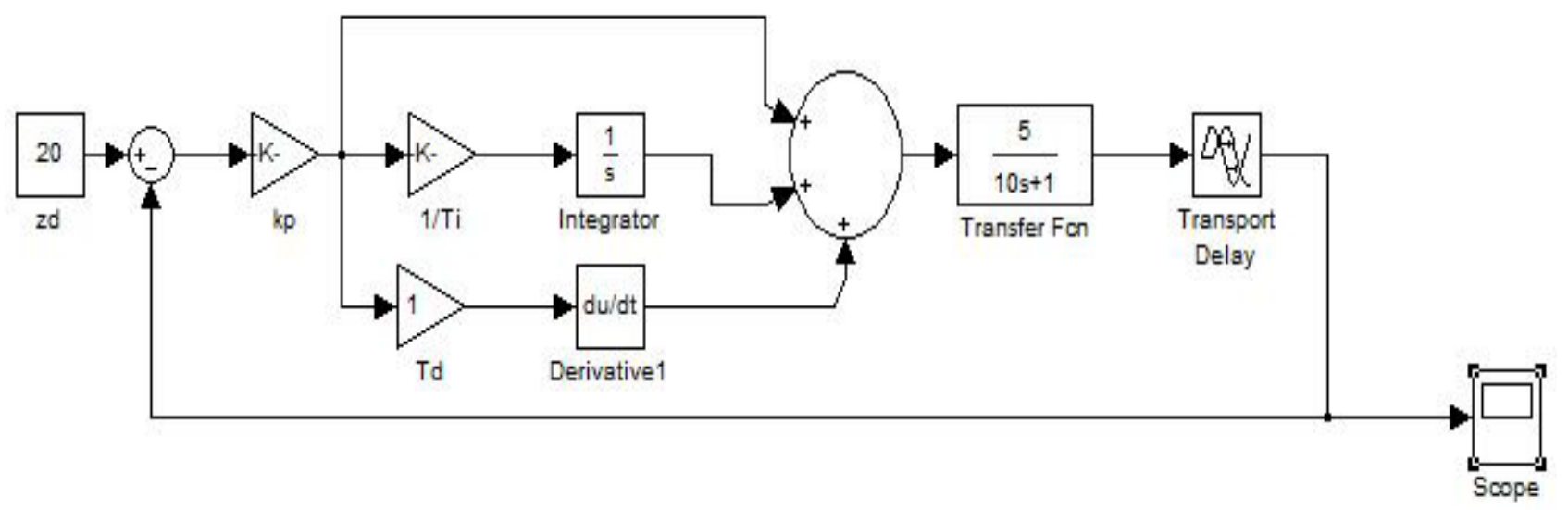

Figure 1. PID control system of temperature

PID controller setting parameters are as above, the temperature control simulation and the control effect are as shown below: 


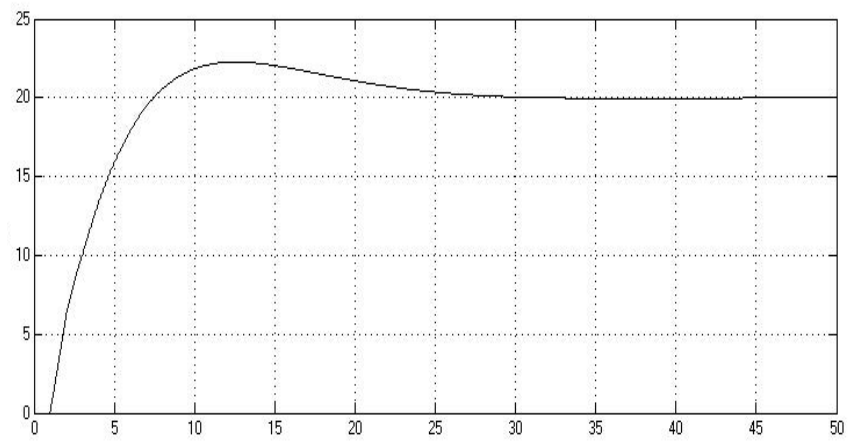

B. Design of differential forward controller for large delay temperature of large-scale chicken farms

In dealing with large delay control problem, when the control requirements are very severe, and conventional PID regulator carefully modulation can not meet the requirements, it can be based on the differential forward control scheme conventional regulator. Differential forward scheme is to move the differential role to the feedback loop to enhance the differential effect, to achieve the effect of reducing the overshoot. Its concrete block diagram is shown in Figure 3:

Figure 2. Simulation result of temperature during PID control

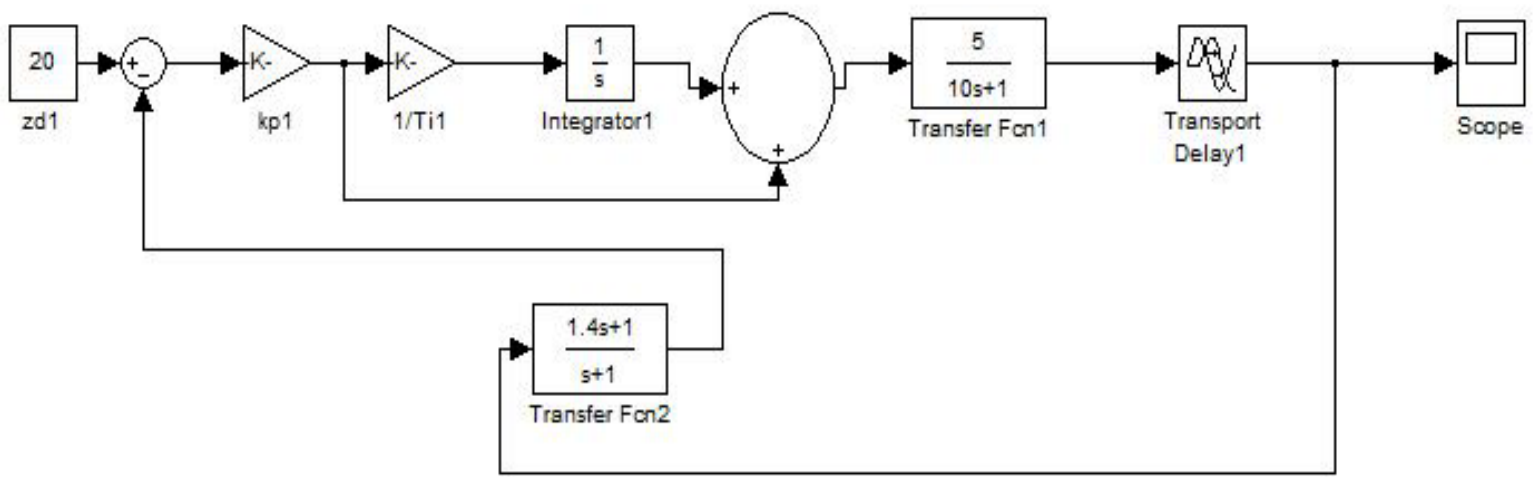

Figure 3. Differential forward control system of temperature

According to the above differential leading controller, and simulation of temperature control, the control effect is as follows:

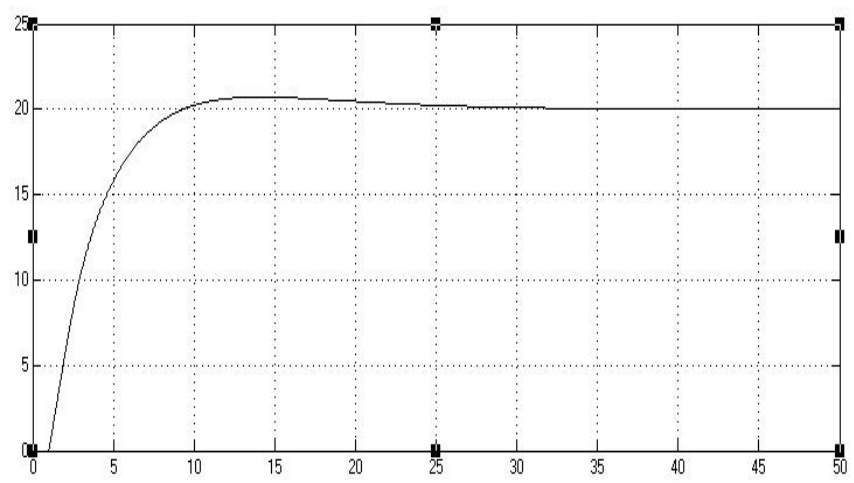

Figure 4. Simulation result of temperature during differential forward control

\section{Smith predictor controller design for large delay temperature of large chicken farm}

In dealing with large time delay control system, the Smith predictor is a very practical method. It is characterized by the dynamic characteristics of the estimated process under the basic disturbance. Then through the predictor compensation, to delay the $\tau$, the amount charged ahead reflected to the regulator, allows the regulator action in advance, thereby significantly reducing the overshoot and accelerate the adjustment process, so fast and accurate response control. Its regulation principle block diagram Figure $5 . \mathrm{K}_{\mathrm{P}} \mathrm{G}_{\mathrm{S}}(\mathrm{s})$ is the Smith predictor compensate, the transfer function is:

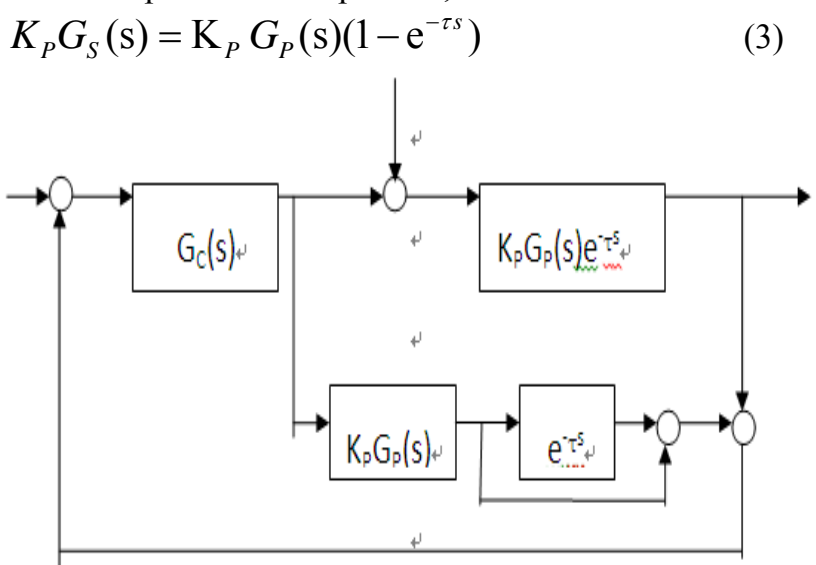

Figure 5. Smith predictor compensator control system of temperature

According to the block diagram of the Smith predictor and the large delay temperature controller model of large chicken farms(1),structural temperature control system simulation diagram is shown in Figure 6.zd in the figure is the desired temperature, here we getting the desired temperature value is 20 degrees, and $\mathrm{K}_{\mathrm{P}}=5 ; \mathrm{T}_{1}=10$ : 


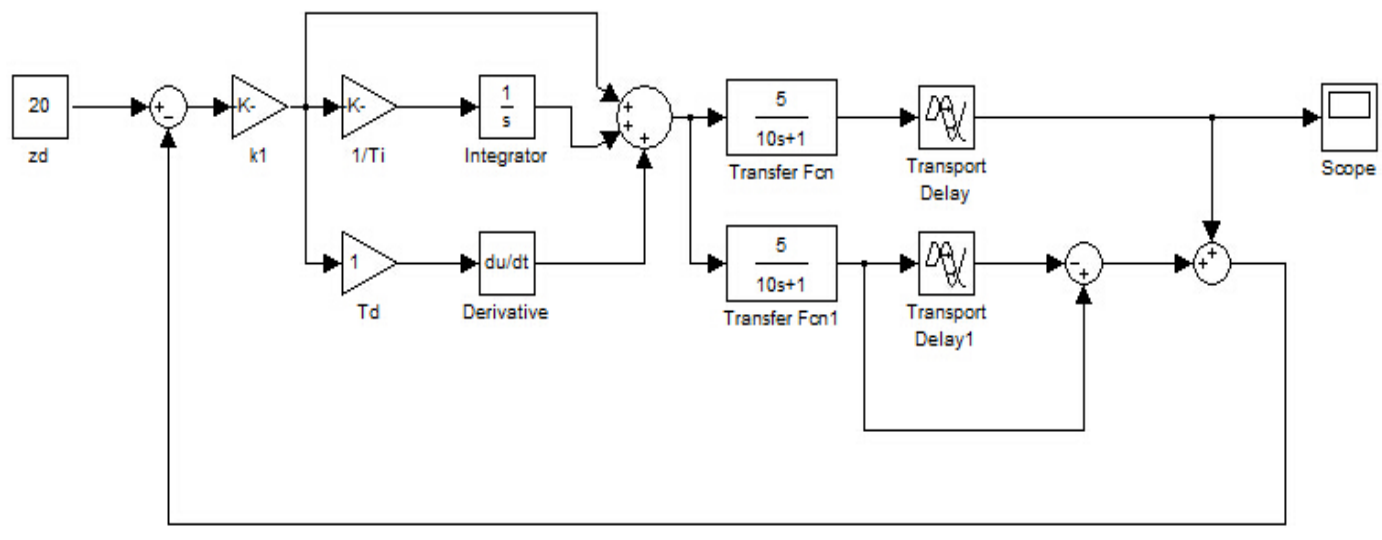

Figure 6. Smith predictor compensator control system of temperature

Setting the Smith predictor compensation parameters and temperature control simulation, get the control effect is as shown below:

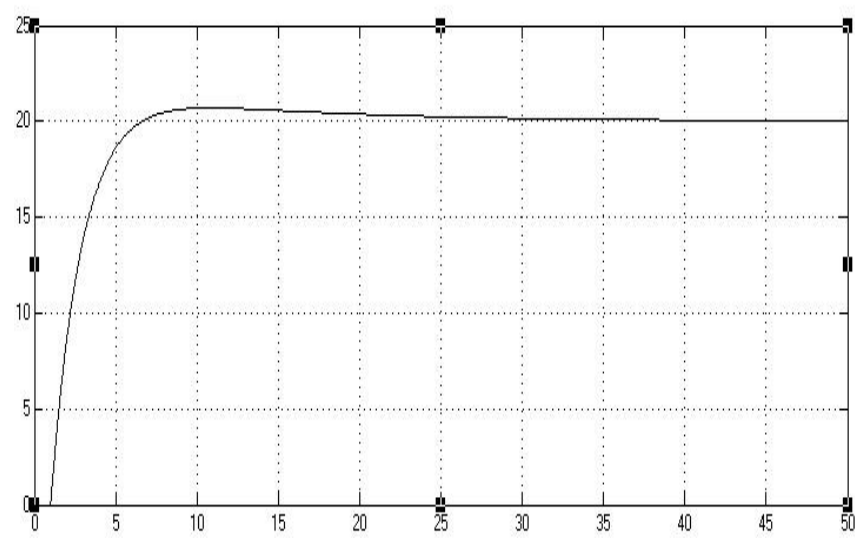

Figure 7. Smith predictor compensator control system of temperature

\section{CONCLUSION}

To build a large chicken field's big delay temperature control model, for the large-scale chicken farms of large time-delay system with conventional PID control, differential advanced control and Smith predictive control method to design the controller and using Simulink simulation, in terms of the big delay system temperature control we can see that the choice of Smith predictive compensator can greatly improve the accuracy and rapidity of the system, and compared with the conventional PID controller, differential first design controller, the Smith predictive compensator method has better rapidity and accuracy, and achieves better control effect.

\section{REFERENCES}

[1] Xiao Ling. The design and implementation of the monitoring platform based on the intelligent terminal[D]. Chang Sha:Central South University of Forestry and Technology.2014.

[2] Zhang Jian. Research and implementation of the intelligent farm field based on Zigbee[D].QuFu: Qufu Normal University.2014.

[3] Gao feng. Lu Shangqiong, Xu Qingxiang et al. Application and development of wireless sensor networks in facility agriculture[J]. Journal of Zhejiang Forestry University,2010,27(5):762-769.

[4] Zhao Meijuan. Research on technology of solar biogas project based on intelligent house[D].BaoDing: Agricultural University of Hebei.2011

[5] Han Jing,Wang Xi,Wang Fuli et al.Research of large-scale cattle farm automatic wireless intelligent environment controller[J]. Journal of Agriculture Mechanization Research,2014,36(12):19-23.

[6] Wang Yang, Fu Zetian, Zhang Lingxian, et al. An Intelligent Ammonia Sensor for Livestock Breeding Monitoring[C]// Computer and Computing Technologies in Agriculture VIIProceeding of 7th IFIP WG 5.14 International Conference. BeiJing, China,2013.

[7] Zhao Liming, Zhang Lanting, Ge Yinsheng, et al.Drinking water equipment[J].China poultry introduction overview.,2001 (24).

[8] Zhao Liming. The installation and commissioning of automatic drinking water equipment and equipment characteristics[J].Chinese poultry.2002(4).

[9] Cao Zhengshu.Automatic drinking water technology for scale pig[J].China animal husbandry and veterinary digest,2011 (6):199200.

[10] Zhang Zhiwei, Wang Zhixi. Selection and maintenance of chicken with drinking water system $[\mathrm{C}] / /$ Proceedings of the second session of the Yellow Feather Broiler Industry Development Conference.Nanning, Guangxi,2010.

[11] Frank Schreurs,Shao Zhenhua, Li Guorong. Smartflow's advantages of intelligent ventilation system in pig farm application[J].Observation of pig,2015(2):71-73.

[12] Li Tielin. The ventilation system in modern poultry farm[J]. China poultryintroduction,2002(12). 